\title{
Mat-O-Covid: Validation of a SARS-CoV-2 Job Exposure Ma- trix (JEM) Using Data from a National Compensation System for Occupational Covid-19
}

\author{
Alexis Descatha ${ }^{1 *}$, Grace Sembajwe ${ }^{2}$, Fabien Gilbert ${ }^{3}$, Mat-O-Covid investigation group ${ }^{* *}$ and Marc Fadel ${ }^{4}$
}

1 Univ Angers, CHU Angers, Univ Rennes, Inserm, EHESP, Irset (Institut de recherche en santé, environnement et travail) - UMR_S 1085, IRSET-ESTER, SFR ICAT, CAPTV CDC, F-49000, Angers, France ; Department of Occupational Medicine, Epidemiology and Prevention, Donald and Barbara Zucker School of Medicine, Hostra University Northwell Health, New York, USA; alexis.descatha@inserm.fr

2 Department of Occupational Medicine, Epidemiology and Prevention, Donald and Barbara Zucker School of Medicine, Hostra University Northwell Health, New York, USA; Sembajwe, Grace N GSembajwe@northwell.edu

3 Univ Angers, CHU Angers, Univ Rennes, Inserm, EHESP, Irset (Institut de recherche en santé, environnement et travail) - UMR_S 1085, IRSET-ESTER, SFR ICAT, CAPTV CDC, F-49000, Angers; fabien.gilbert@univ-angers.fr

4 Univ Angers, CHU Angers, Univ Rennes, Inserm, EHESP, Irset (Institut de recherche en santé, environnement et travail) - UMR_S 1085, IRSET-ESTER, SFR ICAT, CAPTV CDC, F-49000, Angers, France marc.fadel@etud-univ-angers.fr

* Correspondence: alexis.descatha@inserm.fr

** Mat-O-Covid investigation group (list of inverstigator): Alexis Descatha (CHU/ Univ Angers), Marc Fadel (Univ Angers/ AP-HP), Sabrina Pitet (CHU Angers), Fabien Gilbert (Univ Angers), Rémi Valter (AP-HP, Paris), Annette Leclerc (Inserm), Catherine Verdun-Esquer (CHU Bordeaux), Yolande Esquirol (CHU/Univ Toulouse III), Clément Legeay (CHU Angers), Audrey Petit (CHU/ Univ Angers), Aurélien Dinh (AP-HP, Paris Saclay Univ), Pascal Andujar (Univ Paris Est Créteil, Créteil Hospital), Jean-Pierre Leclerc (INRS, Nancy), Corinne Letheux (Presanse, Paris), Pascal Duprat (DIRECCT Ile-de-France, Paris), Brigitte Clodoré (Ville de Paris), Sandrine Cartégnie (SISTBI, La Réunion), Céline Dagrenat (CMIE, Paris), William Dab (CNAM, Paris), Bénédicte Clin-Godard (CHU Caen), Jean-François Gehanno (CHU Rouen), Vincent Dubée (Univ Angers/ CHU Angers), Philippe Havette (La Poste, Paris).

\begin{abstract}
Background. We aimed to assess the validity of the Mat-O-Covid Job Exposure Matrix (JEM) on SARS-CoV2 using compensation data from the French National Health Insurance compensation system for occupational-related COVID-19. Methods. Deidentified compensation data for occupational COVID-19 in France were obtained between August 2020 and August 2021. The acceptance was considered as the reference. Mat-O-Covid is an expert based French JEM on workplace exposure to SARS-CoV2. Bivariate and multivariate models were used to study the association between the exposure assessed by Mat-O-Covid and the reference, as well as the Area Under Curves (AUC), sensitivity, specificity, predictive values, and likelihood ratios. Results. In the 1140 cases included, there was a close association between the Mat-O-Covid index and the reference $(\mathrm{p}<0.0001)$. The overall predictivity was good, with an AUC of 0.78 and an optimal threshold at 13 per thousand. Using Youden's J statistic resulted in 0.67 sensitivity and 0.87 specificity. Both positive and negative likelihood ratios were significant: respectively 4.9 [2.4-6.4] and 0.4 [0.3-0.4]. Discussion. It was possible to assess Mat-O-Covid's validity using data from the national compensation system for occupational COVID-19. Though further studies are needed, Mat-O-Covid exposure assessment appears to be accurate enough to be used in research.
\end{abstract}

Keywords: public health; occupational; Covid; SARS-CoV-2; work; job exposure matrix; JEM; compensation; predictivity; validity; accuracy 


\section{Introduction}

In the context of the Covid-19 pandemic, work seemed to be an important source of exposure, if not the main source of contamination.[1-3] To be able to study work-related SARS-CoV-2, we developed a job exposure matrix (JEM) on SARS-CoV-2: Mat-OCovid.[4,5] After initially validating reliability among experts and correlation with similar, but not identical, data such as $\mathrm{O}^{*} \mathrm{Net}$ and CONSTANCES,[6] we needed to assess its accuracy against a SARS-CoV-2 workplace exposure assessment.

In France, the health insurance system for work-related disorders and injuries has established a national committee for occupational COVID-19 compensation. In these analyses, we aimed to compare the results of the JEM exposure assessment with the conclusions of the first cases of the French COVID committee used as a gold standard.

\section{Materials and Methods}

\subsection{French compensation system}

The French compensation structure for recognizing the occupational nature of a disease is based on two systems:[7]

A list system ('Tables' in French): If the disease is listed as an occupational disease and if the "related conditions" (i.e., diagnosis criteria, time suffering from the condition delay in diagnosis, sometimes the duration of exposure and type of exposure) are met, the disease is presumed to be occupational and the disease is compensated. A new "Table" was created in 2020 which compensated severe COVID-19 cases for salaried workers in the health and social services sectors. This list system can explain why there is a high recognition rate for COVID-19.

A complementary system: If the conditions are not met or if the disease does not appear in the list, compensation may be granted if (1) the victim has a predictable permanent disability rate over $25 \%$; and (2) a committee determines that the disease is directly related to workplace exposure. A national committee was established to study compensation cases of severe COVID-19 for salaried workers in other sectors.

\subsection{Study}

Blind (deidentified) compensation data for occupational COVID-19 in France were obtained between August 2020 and August 2021. The job title was coded using the 2008 International Standard Classification of Occupations (ISCO).

The main outcome was the compensation results of the national committee, i.e., acceptance or rejection. Only rejected cases for lack of exposure were included. Rejections for medical discrepancies, time from end of exposure or medical diagnosis issues were excluded, as well as cases with missing data for job titles.

The Mat-O-Covid JEM used the 'Profession et Catégories Socioprofessionnelles PCS 2003' (French Classification of Occupations) with a metadata crosswalk that transcoded it through ISCO 2008. Mat-O-Covid is an expert-based JEM: a group of four experts in different occupational fields independently coded occupational exposure to SARS-CoV-2 from 0 to 1.[5] Similarly, three other experts coded prevention methods, allowing obtaining a index associated to probability of exposure to SARS-CoV-2 "Mat-O-Covid Index".[6]

The usual statistics were calculated using bivariate analyses (Student T-test, $\mathrm{Chi}^{2}$ ) and multivariate logistic models adjusted for age and sex. Using the compensation results as reference, the sensitivity, specificity, predictive values, likelihood ratio and Area Under Curve (AUC) of the Receiver Operative Characteristic curve were determined. The optimal threshold was calculated using Youden's J statistic. A P-value lower than 0.05 was considered significant and the $95 \%$ confidence interval was calculated. The study was included in the Mat-O-Covid project which was approved by the Ethics Committee of Angers Teaching University Hospital (2021-009), Statistical Analysis System v9.4 (SAS Institute Inc., Cary, NC, USA), and Stata V17.0 SE (StataCorp, Texas 77845, USA). 


\section{Results}

In the first year of France's COVID-19 compensation system for salaried employees, 1140 cases were included with a $95.5 \%$ acceptance rate $(n=1089)$. The mean age was 55.0 years old (+/- 10.0 years, median 57 years old, range $23-84$ years old), and $40 \%$ were women $(n=456)$. The Mat-O-Covid Index was 15.0 per thousand $(+/-10.0$, median 18 , range 0-35per thousand).

There was a close association between the results of the Mat-O-Covid JEM and the Committee's decision, with a mean Mat-O-Covid index of 15.5 per thousand with an acceptance, versus 4.9 per thousand for a refusal $(p<0.0001)$. After adjusting age and sex, the Mat-O-Covid index odds ratio was 1.12 [1.08-1.17] $(\mathrm{p}<0.0001)$.

The overall predictivity was good with an Area Under Curve of 0.78 (Figure 1). The optimal threshold was 13 per thousand using Youden's J statistic, with a sensitivity of 0.67 , a specificity of 0.87 , a low negative predictive value $(0.11)$ and a high positive predictive value (0.99). Both positive and negative likelihood ratios were significant: respectively 4.9 [2.4-6.4] and 0.4 [0.3-0.4]. The association using 13 per thousand $(n=735,64.5 \%)$ was still significant: the Mat-O-Covid odds ratio was $11.5[5.1-26.0](\mathrm{p}<0.0001)$.

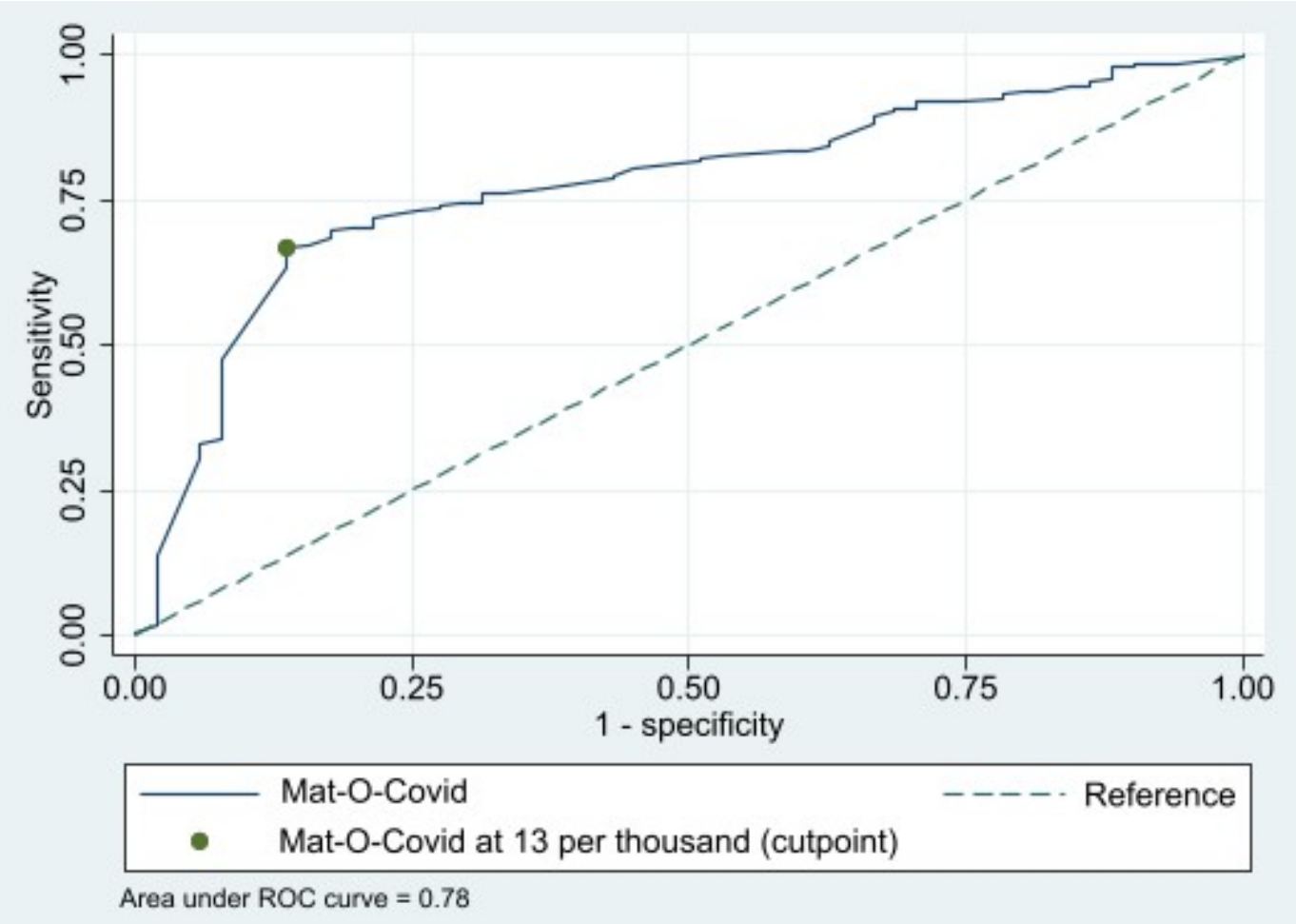

Figure 1. Receiver Operative Characteristic Curve. Mat-O-covid data was compared to the Health Insurance decision on compensation. The Youden's J statistic illustrated the cutpoint.

\section{Discussion}

This study further completed the Mat-O-Covid JEM validation by assessing it against field data. A strong association and fair predictivity against compensation insurance results was found.

It was possible to find a strong association between the Mat-O-Covid JEM and the results of the Committee, and the association is fair. Indeed, the determined threshold (13 per thousand) appears very frequently in the sample, since almost two-thirds of subjects reach it. This is not the only case of a high rate of acceptance in France and there was similar results for musculoskeletal disorders (over $90 \%$ of acceptance).[8] This high acceptance rate has a direct effect on predictive values, but should not influence sensitivity/specificity and likelihood ratios that are robust against variation of prevalence in the 
sample. The low negative predictive value of 361 false negatives is an important limitation when using the Mat-O-Covid JEM on an individual level. Although Mat-O-Covid is a tool for researchers, that might also be used for public health purposes, it is not recommended for individual use.[6,9]

Assessing work-exposure to SARS-CoV2 has become important for research.[10,11] Different study approaches have been developed in other countries based on questionnaire surveys.[12] These surveys are considered more precise in distinguishing task-level differences in exposure among similar job categories, compared to a JEM.[13] However, most large studies don't have specific questions on work, and the JEM would be more accurate when considering asymptomatic cases and estimation of COVID-19, with a lower misclassification bias similar to a chemical JEM.[14] An English working Group developed a control banding matrix to help employers assess the risks of COVID-19 infection during the pandemic, [15] and researchers from Denmark, Netherlands and United Kingdom also developed a JEM defining relevant exposure and workplace characteristics related to exposure to the SARS-CoV2.[16] Differences in country and design would be interesting to compare in future studies.

Some limitations of the JEM approach exist. Only severe cases of COVID-19 can be potentially compensated by the list and complementary system. Public servants and selfemployed individuals are not included in the compensation system. However, less severe cases are similar in public servants and self-employed when it comes to COVID-19 exposure. Second, it is the first validation of workplace exposure while concurrently developing the JEM. Though, this matrix has been found to correlate with previous studies, including $\mathrm{O}^{*} \mathrm{Net}$ (US JEM previously created), external validity is lacking, and further studies are needed with different data that factor time, considering circulating virus and variants. Third, the gold standard was expert judgments and not based on direct measures of exposure data. However, members of the Committee were independent, i.e., not involved in the rating of Mat-O-Covid and blinded to the results of Mat-O-Covid. It allowed us to better assess workplace exposure to COVID-19, in more detail thatn could have been done with direct measures of exposure/contamination data.

\section{Conclusions}

In conclusion, Mat-O-Covid appears to be an accurate assessment of occupational exposure to SARS-CoV-2 that causes COVID-19. Further studies are needed in other work and country settings and that incorporate direct measures of exposure (real world data).

Author Contributions: “Conceptualization, AD.; methodology, AD, GS and MF and MOC Group.; datamanagement, FG.; validation, All authors.; formal analysis, AD.; writing-original draft preparation, AD.; writing - review and editing FG, GS and MFAll authors have read and agreed to the published version of the manuscript."

Funding: This research was funded REACTing Inserm ("Mat-O-Covid project"), ANRS (Emerging Infectious Diseases since 2021), Regional Public Fund ("TEC-TOP project"): Pays-de-la-Loire Region, Angers Loire Métropole, Univ Angers, CHU Angers.

Institutional Review Board Statement: The study was included in the Mat-O-Covid project which was approved by the Ethics Committee of Angers Teaching University Hospital (2021-009),

Informed Consent Statement: Patient consent was waived due to total deidentification and French compensation law.

Data Availability Statement: Data are restricted to French health insurance.

Acknowledgments: We thank the French health insurance for work-related injuries and diseases ("Direction des risques professionnelles de la Caisse Nationale d'Assurance Maladie") for giving permission to access compensation data.

Conflicts of Interest: The authors declare no conflict of interest 


\section{References}

1. Burdorf, A.; Porru, F.; Rugulies, R. The COVID-19 (Coronavirus) Pandemic: Consequences for Occupational Health. Scand. J. Work. Environ. Health 2020, 46, 229-230, doi:10.5271/sjweh.3893.

2. Billingsley, S.; Brandén, M.; Aradhya, S.; Drefahl, S.; Andersson, G.; Mussino, E. COVID-19 Mortality across Occupations and Secondary Risks for Elderly Individuals in the Household: A Population Register-Based Study. Scand. J. Work. Environ. Health 2022, 48, 52-60, doi:10.5271/sjweh.3992.

3. Nafilyan, V.; Pawelek, P.; Ayoubkhani, D.; Rhodes, S.; Pembrey, L.; Matz, M.; Coleman, M.; Allemani, C.; Windsor-Shellard, B.; van Tongeren, M.; et al. Occupation and COVID-19 Mortality in England: A National Linked Data Study of 14.3 Million Adults. Occup. Environ. Med. 2021, oemed-2021-107818, doi:10.1136/oemed-2021-107818.

4. Fadel, M.; Salomon, J.; Descatha, A. COVID-19 Job Exposure Matrix: From the Mat-O-Covid Design to Its Execution. J. Occup. Environ. Med. 2021, 63, e168, doi:10.1097/JOM.0000000000002148.

5. Descatha, A.; Fadel, M.; Pitet, S.; Verdun-Esquer, C.; Esquirol, Y.; Legeay, C.; Dinh, A.; Clodore, B.; Duprat, P.; Cartégnie, S.; et al. SARS-CoV-2 (COVID-19) Job Exposure Matrix: "Mat-O-Covid" Creation (COVID-Mate in French), accuracy study, and perspectives. Arch. Mal. Prof. Environ. 2021, doi:10.1016/j.admp.2021.07.008.

6. Descatha, A.; Pitet, S.; Badreau, M.; Gilbert, F.; Sembajwe, G.; Mat-O-Covid liste Mat-O-Covid: How to Use It? Arch MalProf Env. 2022, in press.

7. Choudat, D. Peut-on Accroître La Cohérence Des Avis Pour La Reconnaissance En Maladie Professionnelle? Arch. Mal. Prof. Environ. 2006, 67, 485-487, doi:10.1016/S1775-8785(06)70388-5.

8. Fadel, M.; Valter, R.; Quignette, A.; Descatha, A. Usefulness of a Job-Exposure Matrix “MADE” as a Decision Tool for Compensation of Work-Related Musculoskeletal Disorders. Eur. J. Public Health 2019, 29, 868-870, doi:10.1093/eurpub/cky274.

9. Descatha, A.; Fadel, M.; Sembajwe, G.; Peters, S. Using the COVID-19 Job Exposure Matrix for Essential Workplace Preparedness. J. Occup. Environ. Med. 2022, 64, e39-e40, doi:10.1097/JOM.0000000000002437.

10. Burdorf, A.; Porru, F.; Rugulies, R. The COVID-19 Pandemic: One Year Later - an Occupational Perspective. Scand. J. Work. Environ. Health 2021, 47, 245-247, doi:10.5271/sjweh.3956.

11. Godeau, D.; Petit, A.; Richard, I.; Roquelaure, Y.; Descatha, A. Return-to-Work, Disabilities and Occupational Health in the Age of COVID-19. Scand. J. Work. Environ. Health 2021, 47, 408-409, doi:10.5271/sjweh.3960.

12. Ingram, C.; Chen, Y.; Buggy, C.; Downey, V.; Archibald, M.; Rachwal, N.; Roe, M.; Drummond, A.; Perrotta, C. Development and Validation of a Multi-Lingual Online Questionnaire for Surveying the COVID-19 Prevention and Control Measures Used in Global Workplaces. BMC Public Health 2022, 22, 74, doi:10.1186/s12889-022-12500-w.

13. Peters, S. Although a Valuable Method in Occupational Epidemiology, Job-Exposure -Matrices Are No Magic Fix. Scand. J. Work. Environ. Health 2020, 46, 231-234, doi:10.5271/sjweh.3894.

14. Bouyer, J.; Hémon, D. [Job exposure matrices]. Rev. Epidemiol. Sante Publique 1994, 42, 235-245.

15. Williams, K.; Cherrie, J.W.; Dobbie, J.; Agius, R.M. The Development of a Covid-19 Control Measures Risk Matrix for Occupational Hygiene Protective Measures. Ann. Work Expo. Health 2021, wxab050, doi:10.1093/annweh/wxab050.

16. Oude Hengel, K.M.; Burdorf, A.; Pronk, A.; Schlünssen, V.; Stokholm, Z.A.; Kolstad, H.A.; van Veldhoven, K.; Basinas, I.; van Tongeren, M.; Peters, S. Exposure to a SARS-CoV-2 Infection at Work: Development of an International Job Exposure Matrix (COVID19-JEM). Scand. J. Work. Environ. Health 2022, 48, 61-70, doi:10.5271/sjweh.3998. 